\title{
Clinical and molecular genetic analysis of a new mutation in children with Wolfram syndrome: A case report
}

\author{
QIANQIAN XU* , HUAIYU QU* and SHIHUI WEI \\ Department of Ophthalmology, PLA General Hospital, Beijing 100853, P.R. China
}

Received September 26, 2012; Accepted January 8, 2013

DOI: $10.3892 / \mathrm{mmr} .2013 .1277$

\begin{abstract}
A 12-year-old Chinese girl presented with gradual vision loss and insulin-dependent diabetes mellitus and was suspected to suffer from Wolfram syndrome (WFS). A series of clinical examinations were performed, as well as direct DNA sequencing to screen the entire coding region of the WFS1 gene in the patient's family, including her parents and a brother. Ophthalmological examination revealed counting fingers $/ 10 \mathrm{~cm}$ in the right eye and hand motions $/ 10 \mathrm{~cm}$ in the left eye. Ophthalmoscopical examination identified bilateral optic atrophy without any signs of diabetic retinopathy. A hearing test was performed and revealed that the hearing ability for high frequency sounds was decreased. Urinary output in $24 \mathrm{~h}$ was $>5,000 \mathrm{ml}$. In addition, a base substitution at c.2411T $>\mathrm{C}$ (Leu804Pro) in exon 8 was identified which was homozygous with the patient and heterozygous with the healthy parents and the brother. In the present case, a neuroophthalmology consult performed in the early stages of the disease was crucial for early diagnosis. In addition, this case study highlights the importance of performing a hearing test as well as collecting and analyzing 24-h urine output in patients presenting with juvenile diabetes mellitus patients and optic atrophy without any signs of diabetic retinopathy.
\end{abstract}

\section{Introduction}

Wolfram syndrome (WFS) is an autosomal recessive neurodegenerative disorder characterized by early onset diabetes mellitus and progressive optic atrophy in children. Patients typically suffer from diabetes insipidus and deafness. Despite a number of studies reporting the incidence of WFS as extremely rare $(1,2)$, several cases have been reported previ-

Correspondence to: Professor Shihui Wei, Department of Ophthalmology, PLA General Hospital, 28 Fuxin road, Beijing 100853, P.R. China

E-mail: shihui.wei@yahoo.cn

${ }^{*}$ Contributed equally

Key words: Wolfram syndrome, optic nerve disease, early diagnosis, mutation, WFS1 gene ously (1-3). It is possible that the incidence of WFS has been underestimated.

Neuroophthalmologists encounter WFS patients who present with optical nerve atrophy. Understanding the clinical characteristics of the disease is important to prevent misdiagnosis. The prognosis of individuals with WFS is poor and $\sim 70 \%$ of patients have an estimated life span of $<35$ years (4). Therefore, early diagnosis is beneficial to the patient and their family. We have found an extremely limited number of ophthalmologists who are familiar with this disease and there are also relatively few studies published in ocular journals.

The WFS1 and CISD2 (also known as WFS2) genes have been identified in association with WFS. The WFS1 gene is on the short arm of chromosome 4. WFS1-related disorders vary between WFS and WFS1-related low-frequency sensory hearing loss. The WFS1 protein (wolframin) is an integral, endoglycosidase $\mathrm{H}$-sensitive membrane glycoprotein that localizes primarily in the endoplasmic reticulum. Numerous mutations have been found in WFS1, largely in exon 8, including missense, insertion, deletion and splice mutations. Compound heterozygote for 2 missense mutations has also been reported and leads to a relatively mild phenotype (5-7). There is no evidence that deletion, splice and insertion mutations cause more severe symptoms than missense mutations (8). A specific missense mutation is known to cause WFS in homozygotes but only low-frequency sensorineural hearing loss in heterozygous carriers $(9,10)$. In the current study, a case of WFS is discussed following detailed investigation of its biological presentation.

\section{Case report}

Patient and clinical data. A 12-year-old Chinese girl presented with gradual loss of vision in both eyes over $\sim 3$ years. The patient did not report any associated symptoms or history, including pain with eye movement, ocular injury, encephalitis or premature delivery. The patient was also diagnosed with Type 1 diabetes mellitus 7 years ago and inadequately controlled blood sugar levels even with insulin treatment. Her parents were not consanguineous and were healthy. No history of diabetes was known in her family. The study was approved by the ethics committee of PLA general hospital, Beijing, China, and written informed consent was obtained from the patient's family.

Ophthalmological examination was performed and revealed counting fingers $/ 10 \mathrm{~cm}$ in the right eye and hand motions $/ 10 \mathrm{~cm}$ in the left eye. Random amplification of poly- 
Table I. Variations in the WFS1 gene of the family.

\begin{tabular}{|c|c|c|c|c|}
\hline Type of variation & Exon & Nucleotide change & Amino acid change & Status \\
\hline SNP & 6 & c. $684 \mathrm{C}>\mathrm{G}$ & - & - \\
\hline SNP & 8 & c. $997 \mathrm{G}>\mathrm{A}$ & V333I & - \\
\hline SNP & 8 & c. $1185 \mathrm{C}>\mathrm{T}$ & - & - \\
\hline SNP & 8 & c. $1500 \mathrm{C}>\mathrm{T}$ & - & - \\
\hline SNP & 8 & c. $1832 \mathrm{G}>\mathrm{A}$ & R611H & - \\
\hline Missense & 8 & c. $2411 \mathrm{~T}>\mathrm{C}$ & L804P & $\begin{array}{l}\text { I: } 1 \text { heterozygous } \\
\text { I: } 2 \text { heterozygous } \\
\text { II: } 1 \text { homozygous } \\
\text { II: } 2 \text { heterozygous }\end{array}$ \\
\hline SNP & 8 & c. $2433 \mathrm{G}>\mathrm{A}$ & - & - \\
\hline SNP & 8 & c. $2565 \mathrm{~A}>\mathrm{G}$ & - & - \\
\hline
\end{tabular}

WFS1, Wolfram syndrome 1 .
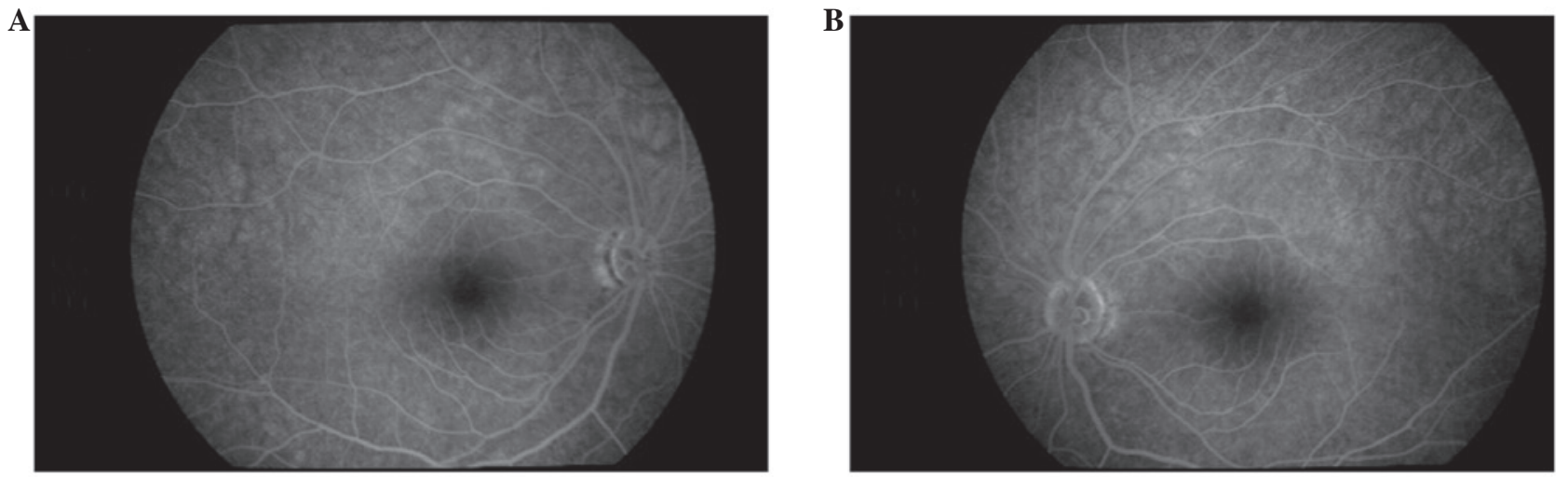

Figure 1. Fundus imaging of the (A) right and (B) left eyes. Images reveal atrophy of the optic nerve only.

morphic DNA was negative in both eyes. Results of intraocular pressure, ocular motility and the anterior segment examination were normal. Ophthalmoscopic examination revealed bilateral optic atrophy without any signs of diabetic retinopathy (Fig. 1). Flash visual-evoked response revealed that the amplitude of P2 decreased. The vision was too poor to perform a visual field test. Magnetic resonance imaging of the brain and spinal cord was normal. Although the patient had no complaint of hearing loss, a hearing test revealed decreased high frequency hearing ability (Fig. 2). Urinary output (24 h) was >5,000 ml. Despite the high urine output, the patient had no complaint of polyuria and considered this output to be normal due to diagnosis of type 1 diabetes mellitus.

Identification of mutations in the WFS1 gene. To detect the presence of WFS1 gene mutations, direct DNA sequencing was performed to screen the entire coding region of the gene in the patient's family (Invitrogen Life Technologies, Beijing, China), including the father, mother and brother. A base substitution at c. $2411 \mathrm{~T}>\mathrm{C}$ (Leu804Pro) was identified in exon 8 which was homozygous with the patient and heterozygous with the healthy parents and brother of the patient (Fig. 3 and Table I).

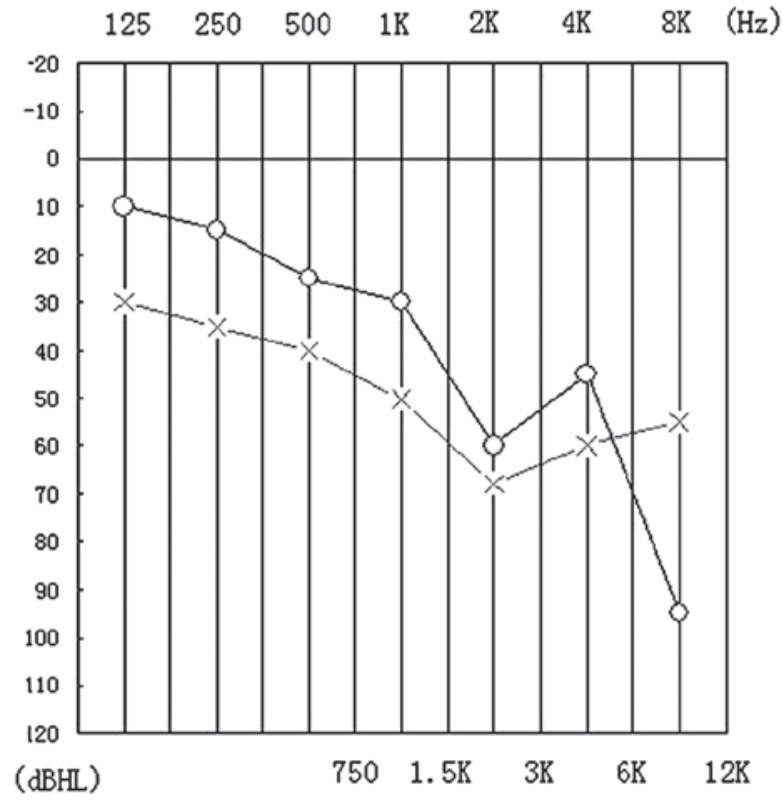

Figure 2. Hearing test revealed the hearing ability of high frequency was decreased. Upper line indicates bone conduction audiometry. Lower line indicates air conduction audiometry. 


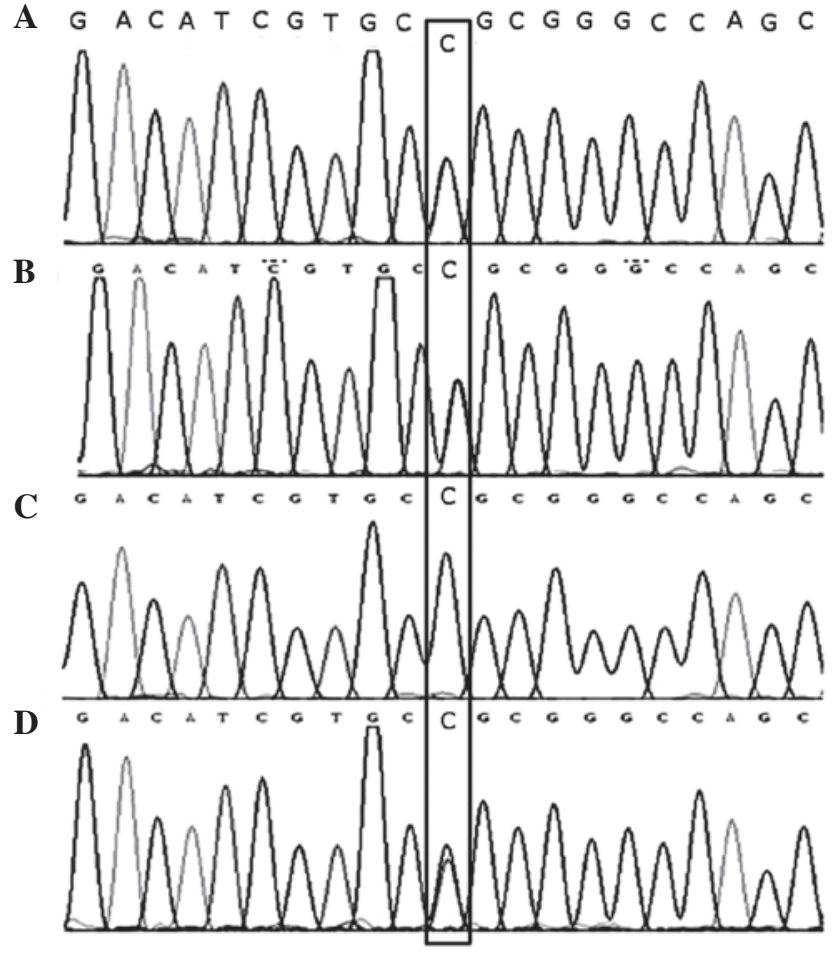

Figure 3. Sequence chromatograms of the region of exon 8 demonstrating the missense at c.2411T>C. (A, B and D) I:1, I:2, II:2, heterozygous for the mutation and $(\mathrm{C}) \mathrm{II}: 1$ homozygous for the mutation.

\section{Discussion}

Wolfram syndrome (WFS) often begins with type 1 diabetes. As there are no other early symptoms, diagnosis is extremely difficult for endocrinologists. As the disease develops, the patient may become aware of worsening vision 2-3 years following diagnosis, due to atrophy of the optic nerve. Patients are often referred to a neuroophthalmologist whose role is crucial to the early diagnosis of this disease.

WFS patients often present with 4 common symptoms. The first is insulin-dependent diabetes mellitus, which is often the first sign of the disease, occuring primarily in childhood. The mean age of onset is $\sim 5-7$ years old $(5,6)$. The current patient was diagnosed with insulin-dependent diabetes mellitus at 5 years old. The second symptom is ocular pathology which often occurs 2-3 years following diagnosis of diabetes. Almost $100 \%$ of WFS individuals present with apparent optic nerve atrophy and additional eye symptoms include visual loss or field defects which may occur earlier than optic nerve atrophy. The patient in this case suffered from considerable optic nerve atrophy with a history of worsening vision loss over 3 years. In addition, diabetes insipidus of hypothalamic origin may occur in the third decade of life in up to $75 \%$ of cases (7). Urinary output (24 h) is often 3-12 liters and may be reduced by administration of an anti-diuretic hormone. Diagnosis of pituitary diabetes insipidus is performed upon additional signs of elevated urine-specific gravity and osmotic pressure. Since individuals with diabetes mellitus commonly suffer from polyuria, WFS patients often consider this output to be normal, however, the high urine volume is actually caused by diabetes insipidus. In the current case, patient 24-h urinary output exceeded 5,000 $\mathrm{ml}$, however, the individual had no complaint of polyuria. Finally, hearing impairment is commonly noted at early stages of WFS. Since the impairment is commonly at high frequency levels of hearing and the frequency range of sounds in everyday life is between 500 and $2,000 \mathrm{~Hz}$, patients often have no complaints of a hearing defect. This was the case in the present WFS individual. The common characteristics of this syndrome demonstrate the importance of performing examination of urine output and a hearing test in patients presenting with insulin-dependent diabetes mellitus and optic nerve atrophy.

It is important for neuroophthalmologists to be able to differentiate WFS from Leber's hereditary optic atrophy and diabetic optic neuropathy, as the prognosis is significantly different. In the case of WFS, $70 \%$ of patients are likely to succumb to the disease prior to 35 years old $(4,11)$. Additional methods, including genetic tests may also aid diagnosis.

Previously, Amr et al (12) revealed that the CISD2 gene, which maps to chromosome $4 \mathrm{q} 22-\mathrm{q} 25$, was localized to the endoplasmic reticulum, colocalizing with calnexin. The authors identified a mutation in the CISD2 gene in 3 consanguineous families of Jordanian descent with WFS. The patient developed renal failure, diabetes mellitus, optic atrophy and high-frequency sensorineural hearing loss.

A genetic mutation associated with the mitochondria and WFS has been reported in a single study. Rotig et al (13) reported a patient who was diagnosed with type 1 diabetes mellitus at 1 year old. During her sixth year the individual gradually developed failure to thrive, cerebellar ataxia, night blindness, progressive external ophthalmoplegia, extrapyramidal syndrome and mental retardation with elevated protein levels in the cerebral spinal fluid (1-1.5 g/l; normal, <0.30) and low-density areas in peduncles and putamen upon nuclear magnetic resonance examination of the brain. The authors identified a 7.6-kb heteroplasmic deletion of mitochondrial DNA in the patient. By contrast, in a previous study of 6 Spanish families with a total of 7 WFS patients, Domenech et al (14) reported no mitochondrial DNA abnormalities (15). Genetic defects in mitochondrial DNA commonly present with several clinical symptoms or a rapidly progressive course with unexplained association of symptoms $(12,16)$.

In the family of the current patient, the parents and brother were heterozygous at c.2411 and did not present with any symptoms of WFS, however, the patient was homozygous and revealed a complete phenotype.

The aim of this case study is to draw attention to the rare disease WFS and highlight the importance of performing a hearing test and analysis of 24-h urinary output when encountering juvenile diabetes mellitus patients with optic atrophy. Genetic tests to identify mutations in WFS1 may also aid diagnosis. In addition, early diagnosis of WFS is likely to aid rapid prognosis prediction. Finally, the differentiation of WFS from diabetic papillopathy and additional diseases caused by trauma, inflammation, tumor or metastasis is extremely important.

\section{References}

1. Barrett TG, Bundey SE and Macleod AF: Neurodegeneration and diabetes: UK nationwide study of Wolfram (DIDMOAD) syndrome. Lancet 346: 1458-1463, 1995.

2. Inukai K, Awata T, Inoue K, et al: Identification of a novel WFS1 mutation (AFF344-345ins) in Japanese patients with Wolfram syndrome. Diabetes Res Clin Pract 69: 136-141, 2005. 
3. Naderian G, Ashtari F, Nouri-Mahdavi K and Sajjadi V: A case of wolfram syndrome. J Ophthalmic Vis Res 5: 53-56, 2010.

4. Fabbri LP, Nucera M, Grippo A, et al: Wolfram syndrome. How much could knowledge challenge the fate? A case report. Med Sci Monit 11: CS40-CS44, 2005.

5. Simsek E, Simsek T, Tekgul S, Hosal S, Seyrantepe V and Aktan G: Wolfram (DIDMOAD) syndrome: a multidisciplinary clinical study in nine Turkish patients and review of the literature. Acta Paediatr 92: 55-61, 2003

6. Medlej R, Wasson J, Baz P, et al: Diabetes mellitus and optic atrophy: a study of Wolfram syndrome in the Lebanese population. J Clin Endocrinol Metab 89: 1656-1661, 2004.

7. Piccoli GB, Mezza E, Jeantet A and Segoloni GP: An uncommon genetic syndrome with acute renal failure in a 30-year-old diabetic patient. Nephrol Dial Transplant 18: 206-208, 2003.

8. d'Annunzio G, Minuto N, D'Amato E, et al: Wolfram syndrome (diabetes insipidus, diabetes, optic atrophy and deafness): clinical and genetic study. Diabetes Care 31: 1743-1745, 2008

9. Hardy C, Khanim F, Torres R, et al: Clinical and molecular genetic analysis of 19 Wolfram syndrome kindreds demonstrating a wide spectrum of mutations in WFS1. Am J Hum Genet 65: 1279-1290, 1999.

10. Young TL, Ives E, Lynch E, et al: Non-syndromic progressive hearing loss DFNA38 is caused by heterozygous missense mutation in the Wolfram syndrome gene WFS1. Hum Mol Genet 10: 2509-2514, 2001.
11. Cryns K, Sivakumaran TA, Van den Ouweland JM, et al: Mutational spectrum of the WFS1 gene in Wolfram syndrome, nonsyndromic hearing impairment, diabetes mellitus and psychiatric disease. Hum Mutat 22: 275-287, 2003.

12. Amr S, Heisey C, Zhang M, et al: A homozygous mutation in a novel zinc-finger protein, ERIS, is responsible for Wolfram syndrome 2. Am J Hum Genet 81: 673-683, 2007.

13. Rotig A, Cormier V, Chatelain P, et al: Deletion of mitochondrial DNA in a case of early-onset diabetes mellitus, optic atrophy and deafness (Wolfram syndrome, MIM 222300). J Clin Invest 91: 1095-1098, 1993.

14. Domenech E, Gomez-Zaera M and Nunes V: Study of the WFS1 gene and mitochondrial DNA in Spanish Wolfram syndrome families. Clin Genet 65: 463-469, 2004.

15. Chaussenot A, Bannwarth S, Rouzier C, et al: Neurologic features and genotype-phenotype correlation in Wolfram syndrome. Ann Neurol 69: 501-508, 2011.

16. Zalloua PA, Azar ST, Delepine M, et al: WFS1 mutations are frequent monogenic causes of juvenile-onset diabetes mellitus in Lebanon. Hum Mol Genet 17: 4012-4021, 2008. 